\title{
BIANCHI COSMOLOGIES AS DYNAMICAL SYSTEMS
}

\author{
ANDRZEJ J. MACIEJEWSKI \\ Toruń Centre for Astronomy, N. Copernicus University, \\ Chopina 12/18,87-100 Toruń, Poland; E-mail: maciejka@astri.uni.torun.pl \\ and \\ MAREK SZYDłOWSKI \\ Astronomical Observatory, Jagiellonian University, \\ Orla 171, 30-244 Kraków, Poland; E-mail: uoszydlo@cyf-kr.edu.pl
}

\begin{abstract}
We discuss specific properties of dynamical systems originating from cosmology and relativity. In particular, we present results of our study of the Bianchi class A cosmological models. We introduce new variables in which the Hamiltonian constraint for all the class A models is solved algebraically. We present results of dimension reduction of the investigated models.
\end{abstract}

\section{Introduction}

Models of relativistic cosmology are based on Einstein's theory of gravitation. The Einstein field equations describe the dynamical evolution of spacetime, as well as the motion of matter and physical fields. They provide a system of coupled, non-linear, partial differential equations. Without some simplifying assumptions or idealization they are intractable by analytical tools. The most natural assumption is to postulate a certain symmetry of space-time. Usually, such idealization allows to reduce Einstein's field equations to a system of ordinary differential equations. This reduction gives us a possibility to use the rich theory of dynamical systems. It has to be mentioned, however, that dynamical systems of cosmological (or relativistic) origin have many special features which distinguish them from a 'typical' dynamical system we meet in dynamical astronomy, classical mechanics or physics. Let us mention a few of them. We can assume that systems we meet have the following form

$$
\dot{x}=v(x), \quad x \in \mathbb{R}^{n} \text {. }
$$

1. Although the right hand sides of (1) are polynomial, in many cases it is not obvious if the phase flow of this system is complete. It seems that in some cases it is not. It is important to point it out, because for systems with an incomplete flow customary indicators of chaos are not defined, although numerical algorithms do not distinguish between complete and incomplete flows.

2. In many cases the system (1) has a first integral $H$. Usually, only solutions lying on the level

$$
\mathcal{M}=\left\{x \in \mathbb{R}^{n} \mid H(x)=0\right\},
$$

have a physical interpretation. Thus we have to restrict our system to an invariant set $\mathcal{M}$. However, in many investigations this point is simply ignored. For example, when we ask for the existence of one or more additional first 
integrals then a negative answer for the non-restricted system is not valid for the system restricted to $\mathcal{M}$.

3. In most cases the equilibria of (1) are degenerated and lie on an one or higher dimensional manifold of equilibria.

4. Frequently the system (1) is Hamiltonian with respect to the canonical symplectic form $\omega$ on $\boldsymbol{R}^{n}, n=2 m$, and the first integral $H$ plays the role of the Hamiltonian function, i.e., $\omega(v, \cdot)=d H$. Usually, the Hamiltonian has the 'natural' form

$$
H=\frac{1}{2} g^{i j} p_{i} p_{j}+V(q), \quad x=\left(q^{1}, \ldots, q^{m}, p_{1}, \ldots, p_{m}\right) \in \mathbb{R}^{2 m},
$$

however, the 'kinetic' energy $T=\frac{1}{2} g^{i j} p_{i} p_{j}$ is not positive definite. Because of this, there exists no such notion as 'region of possible motion' which plays a fundamental role in studies of natural mechanical systems in classical mechanics. It seems that systems with indefinite kinetic energy possess their own specific properties, however, investigation of these systems is far from being complete.

The last decades gave an immense popularity to the notion of deterministic chaos. Thus, it was natural to look for this phenomenon in the systems mentioned. However, a lot of controversies arose around this subject. On the one hand, these disputes were connected with the numerical character of the obtained results, and, on the other hand, they were caused by some conceptual problems. Here we point out some aspects of this discussion showing our point of view.

The main example of our discussion are class A Bianchi cosmological models. In fact, the controversy about chaotic or non-chaotic behavior concentrates around Bianchi IX model. We describe these models in the next section. In Section 3 we present our original results connected with reduction and simplification of class $A$ Bianchi dynamical systems.

\section{Bianchi Class A Cosmologies as Dynamical Systems}

Let us assume that space-time has a product topology of type $\mathbb{R} \times \mathcal{M}^{3}$, where $\mathcal{M}^{3}$ is 3-dimensional space-like section admitting the action of simply transitive isometry group, i.e. homogeneity group; then Einstein's equations take the form of a system of ordinary differential equations. The classification of all 3-dimensional homogeneous but anisotropic spaces according to the Lie algebra of the isometry group is called the Bianchi classification (Landau and Lifshitz, 1975).

In this contribution we consider only subclass A of all Bianchi types for which, without any loss of generality, one can assume that the metric of $\mathcal{M}^{3}$ is diagonal, i.e.

$$
d s^{2}=(a b c) d \tau^{2}-\eta_{a b}(t)\left(e_{i}^{a} d x^{i}\right)\left(e_{j}^{b} d x^{j}\right),
$$

where

$$
\eta_{a b}=\operatorname{diag}\left\|a^{2}(t), b^{2}(t), c^{2}(t)\right\|, \quad a, b=1,2,3,
$$


$\omega^{a}=e_{i}^{a} d x^{i}$ form the base of invariant 1 -forms depending only on spatial variables $\left(x^{i}\right)=(x, y, z)$, while $\eta_{a b}$ is a symmetric metric tensor depending only on the cosmological time $t: d t=a b c d \tau ; \tau$ is called the synchronic time.

The $(a, b)$ components of Einstein's field equations

$$
R_{k}^{i}=0 \text {, }
$$

for our case give

$$
\begin{aligned}
2(\log a)_{\tau \tau} & =\left(n_{2} b^{2}-n_{3} c^{2}\right)^{2}-n_{1}^{4} a^{4}, \\
2(\log b)_{\tau \tau} & =\left(n_{1}^{2} a^{2}-n_{3}^{2} c^{2}\right)^{2}-n_{2}^{4} b^{4}, \\
2(\log c)_{\tau \tau} & =\left(n_{1}^{2} a^{2}-n_{2}^{2} b^{2}\right)^{2}-n_{3}^{4} c^{4},
\end{aligned}
$$

where $n_{i} \in\{0,+1,-1\}$ distinguish the Bianchi type of model. From the $(0,0)$ component $R_{0}^{0}=0$ we obtain

$$
\frac{1}{2}(\log a b c)_{\tau \tau}=(\log a)_{\tau}(\log b)_{\tau}+(\log a)_{\tau}(\log c)_{\tau}+(\log b)_{\tau}(\log c)_{\tau} .
$$

After a simple manipulation, we obtain from (5) and (6) the following identity

$$
H=H\left(a, b, c, a_{\tau}, b_{\tau}, c_{\tau}\right)=0,
$$

where $H$ is first integral of (5) of the form

$$
\begin{array}{r}
H=(\log a)_{\tau}(\log b)_{\tau}+(\log a)_{\tau}(\log c)_{\tau}+(\log b)_{\tau}(\log c)_{\tau} \\
+\frac{1}{4}\left(n_{1}^{4} a^{4}+n_{2}^{4} b^{4}+n_{3}^{4} c^{4}-2 n_{1}^{2} n_{2}^{2} a^{2} b^{2}-2 n_{1}^{2} n_{3}^{2} a^{2} c^{2}-2 n_{2}^{2} n_{3}^{2} b^{2} c^{2}\right) .
\end{array}
$$

The system (5) forms fundamental dynamical equations for the evolution of three different scale factors $a, b, c$ in three different main directions. In the special case when $a=b=c$ equations (5) represent the Friedmann-Robertson-Walker (FRW) models. Therefore, the Bianchi models are more general than the FRW models (standard models of current cosmology), because we assume only homogeneity of space-like sections. There are different reasons to analyze the evolution of cosmology models which are more general than FRW models (see Wainwright and $\mathrm{Hsu}, 1989$ for details) but the main problem is to find a more realistic description of the very early evolution of universe which leads to the presently observed galactic epoch.

Now, we transform the system (5) to a set of first order homogeneous polynomial equations. To this end let us introduce new variables

$$
y_{1}=a^{2}, \quad y_{2}=b^{2}, \quad y_{3}=c^{2}, \quad z_{i}=\frac{\dot{y}_{i}}{y_{i}} .
$$

After this change of variables the system (5) reads

$$
\dot{y}_{i}=y_{i} z_{i}, \quad \dot{z}_{i}=\left(n_{j} y_{j}-n_{k} y_{k}\right)^{2}-n_{i}^{2} y_{i}^{2},
$$


where a dot denotes differentiation with respect to $\tau$. In the new variables first integral (7) has the form

$$
H=\sum_{i=1, i<j}^{3}\left(z_{i} z_{j}+2 n_{i} n_{j} y_{i} y_{j}\right)-\sum_{i=1}^{3} n_{i}^{2} y_{i}^{2} .
$$

The basic problem is to investigate qualitative properties of the above system.

Dynamical systems methods were first applied in the study of the Bianchi cosmologies by Collins (1971) who considered a number of special cases in which the phase space was two-dimensional. The systematic approach to the study of dynamics of all Bianchi cosmological models was initiated by Bogoyavlenski (1985) who investigated their evolution in 5-dimensional phase space. Another description of the dynamics of Bianchi class A models as a dynamical system was presented by Wainwright and Hsu (1989). They used expansion-normalized variables and an orthonormal frame approach (for details see Wainwright and Ellis, 1997).

There exist several directions in which we can start our study. It seems, however, that the most interesting problems can be extracted from studies of Bianchi IX (Mixmaster) model, for which $n_{1}=n_{2}=n_{3}=1$. The numerically computed maximal Lyapunov exponent for this system was zero or different from zero depending on the time parametrisation. Because an approximation of the Mixmaster model by a discrete map has strong chaotic properties (Chernoff and Barrow, 1993), it was natural to expect such a behavior in the original system. However, as Cushman and Śniatycki (1995) proved, there exists no recurrence in the system and, thus, no form of standard deterministic chaos is present in it. Interesting numerical works (Cornish, 1997; Cornish Levin, 1997), where 'parametrisation independent' characteristics of chaos were used, do not make big progress in understanding the system. The point is that in these works a certain approximation of the Mixmaster model was investigated, not the Mixmaster model itself. Moreover, one notices several very unprecise notions used in these investigations.

Because a strict proof of chaotic (in a certain sense) behavior of this system seems very difficult, several authors tried to show that the Mixmaster model is not integrable. It must be mentioned that 'integrability' here was understood differently by different authors. Several authors tested if the model passes the standard Painleve integrability test in the form of the ARS algorithm (Ramani et al., 1989). Contopoulos et al. (1993) indicated that the B(IX) model passes this test. This result was revised (Contopoulos et al., 1994), however, without any strict conclusions concerning integrability. Further studies of Latifi et al. (1995) showed that the B(IX) model does not pass the so called perturbative Painlevé test. The authors of this paper suggest the existence of 'some chaotic regimes' in the system. The strongest result in this direction was obtained in (Contopoulos et al., 1995) where the authors show the existence of movable critical essential singularities in the $\mathrm{B}(\mathrm{IX})$ model. This kind of investigations connect a complicated behavior of the 
system with singularities of their solutions on the complex time plane. It should be mentioned, however, that the relation between Painlevés test and integrability, e.g. in the Liouville's sense, is not clear. One can notice also that the integrability problem was stated for the global system, not for its restriction to the level $H=0$.

The strongest and mathematically precise result concerning the Mixmaster model was obtained by J.J. Morales-Ruiz and J.-P. Ramis on the base of their theory connecting Ziglin's method and differential Galois theory (Morales-Ruiz and Ramis, 1997). We state this result shortly. The Mixmaster model can be formulated as a Hamiltonian system. There exists a four dimensional invariant manifold $\mathcal{I}$ on which the system can be integrated explicitly. Solutions of the system restricted to $\mathcal{T}$ are known as Taub solutions. Studying variational equations around Taub solutions J.J. Morales-Ruiz and J.-P. Ramis proved that the Mixmaster model considered as a complex Hamiltonian system is not completely integrable (in the Liouville sense) with rational first integrals.

We make a few remarks about these results. First, it was not excluded that this system possesses one addition rational integral or is integrable in terms of nonrational integrals. Moreover, it can possess an additional integral only on the level $H=0$ or it can be integrable on it. Thus, by no means, the result of Morales-Ruiz and Ramis closes the subject.

From the above it follows that investigations of system (9) need strong and precise tools, and that the most interesting questions are connected with (partial) integrability of this system.

Although the Mixmaster model seems to be the most attractive, we decided to analyze all class A Bianchi models. Models from this class which have a regular behavior can be used to approximate the complex behavior of Mixmaster models (Belinskii et al., 1982). It will be shown that the dynamics of the B(I) and B(II) models can be represented on a two dimensional phase space, whereas for the $\mathrm{B}\left(\mathrm{VI}_{0}\right)$ and the $\mathrm{B}\left(\mathrm{VII}_{0}\right)$ dynamical systems are three dimensional. The dynamics of these systems can be described precisely.

\section{The Bianchi Class A Models in the Reduced Form}

The main idea that we propose to apply for the study of the Bianchi class A models is the following. The right hand sides of the system (9) are homogeneous polynomials of degree two. Let us denote the vector field connected with them by $X$. The homogeneity of the system can be considered as its certain generalized symmetry. In fact, if we introduce the Euler vector field

$$
E=\sum_{i=1}^{3} y_{i} \frac{\partial}{\partial y_{i}}+z_{i} \frac{\partial}{\partial z_{i}},
$$

then it can be shown that

$$
L_{E} X:=[E, X]=X,
$$


where $L_{E}$ denotes the Lie derivative along the vector field $E$, and $[\cdot, \cdot]$ denotes the Lie bracket. This symmetry can be used to lower the dimension of the system. Moreover, we want to restrict explicitly the system to the zero level of first integral $H=0$, because of the physical interpretation of our system. We will also try to preserve the polynomial form of the right hand sides (Maciejewski and Szydłowski, 1998).

We start from the following change of variables

$$
u_{1}=z_{1} / w_{3}, \quad u_{2}=z_{2} / w_{3}, \quad u_{3}=z_{3} / w_{3}, \quad u_{4}=w_{2} / w_{3}, \quad u_{5}=w_{3},
$$

where

$$
w_{1}=y_{1}+y_{2}, \quad w_{2}=y_{1}-y_{2}, \quad w_{3}=y_{3} .
$$

Then the system (9) has the form

$$
\begin{aligned}
\frac{d u_{1}}{d s} & =p\left(u_{3}, u_{4}, u_{5}\right)-2 u_{1} u_{3}, \\
\frac{d u_{2}}{d s} & =-p\left(u_{3}, u_{4}, u_{5}\right)-2 u_{2} u_{3}, \\
\frac{d u_{3}}{d s} & =\frac{1}{2}\left\{\left[\left(n_{2}-n_{1}\right) u_{5}-\left(n_{1}+n_{2}\right) u_{4}\right]^{2}-4 n_{3}^{2}-4 u_{3}^{2}\right\}, \\
\frac{d u_{4}}{d s} & =\left(u_{1}+u_{2}-2 u_{3}\right) u_{4}+\left(u_{1}-u_{2}\right) u_{5}, \\
\frac{d u_{5}}{d s} & =\left(u_{1}+u_{2}-2 u_{3}\right) u_{5}+\left(u_{1}-u_{2}\right) u_{4}, \\
\frac{d u_{6}}{d s} & =2 u_{3} u_{6},
\end{aligned}
$$

where

$$
\begin{aligned}
p\left(u_{3}, u_{4}, u_{5}\right)= & \frac{1}{2}\left[\left(n_{2}-n_{1}\right) u_{5}+\left(-n_{1}-n_{2}\right) u_{4}-2 n_{3}\right] \times \\
& {\left[\left(n_{1}+n_{2}\right) u_{5}+\left(n_{1}-n_{2}\right) u_{4}-2 n_{3}\right], }
\end{aligned}
$$

and $d s=\frac{1}{2} u_{6} d \tau$.

Let us notice that in equations (12-16) the variable $u_{6}$ does not appear explicitly, i.e. the dynamical system (12-17) separates into two subsystems, from which system (12-16) is closed. The time dependence of the variable $u_{6}$ is determined by integration of the equation (17)

$$
u_{6}=u_{06} \exp \int^{\tau} u_{3}\left(\tau^{\prime}\right) d \tau^{\prime}
$$

Therefore instead of studying the 6-dimensional system (12-17) one can study only the system (12-16) because the full information about the dynamics is contained in the reduced system. Thus, our system has five 'true degrees of freedom'. 


\section{TABLE I}

The collection of constants $n_{i}$ and the respective forms of the first integral for Bianchi A cosmological models. $k$ denotes the dimension of the reduced system.

\begin{tabular}{c|c|c|c|l|l} 
Bianchi type & $n_{1}$ & $n_{2}$ & $n_{3}$ & first integral & $k$ \\
\hline B I & 0 & 0 & 0 & $H=\sum_{i<j}^{3} u_{i} u_{j}$ & 2 \\
B II & 1 & 0 & 0 & $H=4 \sum_{i<j}^{3} u_{i} u_{j}-u_{5}^{2}-u_{4}^{2}-2 u_{4} u_{5}$ & 2 \\
B VI $_{0}$ & 1 & -1 & 0 & $H=\sum_{i<j}^{3} u_{i} u_{j}-u_{5}^{2}$ & 3 \\
B VII $_{0}$ & 1 & 1 & 0 & $H=\sum_{i<j}^{3} u_{i} u_{j}-u_{4}^{2}$ & 3 \\
B VIII & 1 & 1 & -1 & $H=\sum_{i<j}^{3} u_{i} u_{j}-u_{4}^{2}-2 u_{5}-1$ & 4 \\
B IX & 1 & 1 & 1 & $H=\sum_{i<j}^{3} u_{i} u_{j}-u_{4}^{2}+2 u_{5}-1$ & 4
\end{tabular}

Moreover, the system (12-17) has the first integral

$$
\begin{aligned}
H= & \sum_{i<j}^{3} u_{i} u_{j}-\frac{1}{4}\left(n_{1}-n_{2}\right) u_{5}^{2}-\frac{1}{4}\left(n_{1}+n_{2}\right)^{2} u_{4}^{2}-n_{3}^{2} \\
& +\frac{1}{2}\left(n_{2}^{2}-n_{1}^{2}\right) u_{4} u_{5}+\left(n_{1} n_{3}+n_{2} n_{3}\right) u_{5}+\left(n_{1} n_{3}-n_{2} n_{3}\right) u_{4},
\end{aligned}
$$

which in turn can be used to reduce the dimension of dynamical system by one.

Table 1 contains the forms of first integral (19) for all Bianchi class A types. It is easy to see that in all cases one variable can be eliminated, for example in the most general case of the Mixmaster models (B(IX) and $\mathrm{B}(\mathrm{VIII})$ ) it is possible to eliminate the variable $u_{5}$ if we take into account that

$$
u_{5}=\frac{1}{2} n_{3}\left(u_{4}^{2}+1-\sum_{i<j}^{3} u_{i} u_{j}\right),
$$

on the level $H=0$. For these two systems four equations (12-15) with $u_{5}$ defined by (20) form a closed system. It defines the reduced dynamics for the B(IX) and B(VIII) models. An important outcome of this reduction is the fact that we are able to prove that neither of these systems (considered on $\mathbb{C}^{4}$ ) has an additional analytic first integral. A proof of this fact for the B(IX) model can be found in our paper (Maciejewski and Szydłowski, 1998). For the B(VIII) model the proof is similar.

For the rest of the class A Bianchi models we can perform a next step of reduction. Here we present only some basic facts. Details and complete analysis of the reduced systems will be published elsewhere.

For the Bianchi I model, the first three of equations (12-17) can be integrated explicitly. The last two after redefinition of the time variable $u_{1} d s=d \bar{s}$ can be written in the form of two linear differential equations depending on two parameters. Thus, we can perform a complete analysis of this system. 
For the Bianchi II model three equations separate from the rest and they have the following form

$$
\begin{aligned}
\frac{d u_{1}}{d s} & =-2 K\left(u_{1}, u_{2}, u_{3}\right)-2 u_{1} u_{3}, \\
\frac{d u_{2}}{d s} & =2 K\left(u_{1}, u_{2}, u_{3}\right)-2 u_{2} u_{3}, \\
\frac{d u_{3}}{d s} & =2 K\left(u_{1}, u_{2}, u_{3}\right)-2 u_{3}^{2},
\end{aligned}
$$

where $K\left(u_{1}, u_{2}, u_{3}\right)=\sum_{i<j}^{3} u_{i} u_{j}$. Because the right-hand sides of the above system are homogeneous functions of order two, we can introduce the projective variables and reduce it to a two dimensional system, which can be integrated explicitly.

For the Bianchi $\mathrm{VI}_{0}$ and $\mathrm{BVII}_{0}$ models the reduction leads to a three dimensional system of the form

$$
\begin{aligned}
& \frac{d x}{d \bar{s}}=-x^{2}+(z+1) y^{2}, \\
& \frac{d y}{d \bar{s}}=-4(z+1)+x y z, \\
& \frac{d z}{d \bar{s}}=-y z(z+2) .
\end{aligned}
$$

This system has two two-dimensional invariant manifolds $z=0$ and $z=-2$. On these manifold we can perform explicit integration. It is unclear if the system $(24-26)$ is integrable or not.

\section{References}

L.D. Landau and E.M. Lifshitz: 1975, The Classical Theory of Fields. Pergamon, Oxford.

J. Wainwright and G.F.R. Ellis: 1997,Dynamical Systems in Cosmology, Cambridge U.P., Cambridge.

C. B. Collins: 1971 Commun. Math. Phys., 23, 137 (1971).

O.I. Bogoyavlensky: 1985, Methods in Qualitative Theory of Dynamical Systems in Astrophysics and Gas Dynamics. New York, Springer-Verlag.

J. Wainwright and L. Hsu: 1989 Class. Quantum Grav., 6, 1409.

D. F. Chernoff and J. D. Barrow: 1983, Phys. Rev. Lett., 20, (1983), 134-137.

R. Cushman and J. Śniatycki: 1995, Preprint Univ. of Calgary, .

N.J. Cornish: 1997, in Proceedings of Eight M. Grossmann Meeting, Jerusalem.

N.J. Cornish and J.J. Levin: 1997, in Proceedings of Eight M. Grossmann Meeting, Jerusalem.

A. Ramani, B. Grammaticos and T. Bountis: 1989, Phys. Reports 180, 159-254.

G. Contopoulos, B. Grammaticos and A. Ramani: 1993, J. Phys., A26, 5795.

G. Contopoulos, B. Grammaticos and A. Ramani: 1994, J. Phys. A, 27, 5357-5361.

A. Latifi, M. Musette and R. Conte: 1995, Phys. Lett. A, 194, 83-97.

G. Contopoulos, B. Grammaticos and A. Ramani: 1995, J. Phys. A, 28, 5313-5322.

J.J. Morales-Ruiz and J.-P. Ramis: 1997, Galoisian Obstruction to Integrability of Hamiltonian Systems I, II, Preprint.

V. A. Belinskii, I. M. Khalatnikov and E. M. Lifshitz: 1982, Adv. Phys., 31, 639.

A. J. Maciejewski and M. Szydłowski: 1998, J. Phys. A. Math. \& Gen., 31, 2031. 